\title{
Pentacyclic Triterpenoids from Leaves of Excoecaria agallocha
}

\author{
Jian-Hua Zou, ${ }^{a, b}$ Jungui DAI, ${ }^{*, a, b}$ Xiaoguang CHEN, ${ }^{a, b}$ and Jing-Quan YUAN ${ }^{c}$ \\ ${ }^{a}$ Key Laboratory of Bioactive Substances and Resources Utilization of Chinese Herbal Medicine (Peking Union Medical \\ College), Ministry of Education; Beijing, 100050 P.R. China: ${ }^{b}$ Institute of Materia Medica, Chinese Academy of Medical \\ Sciences and Peking Union Medical College; Beijing 100050, P.R. China: and ${ }^{c}$ Guangxi Affiliated Institute of Medicinal \\ Plant Development, Chinese Academy of Medical Sciences \& Guangxi Institute of Medicinal Plant; Nanning 530023, P.R. \\ China. Received February 22, 2006; accepted March 22, 2006
}

A new oleanane-type triterpenoid (1) and five known pentacyclic triterpenoids (2-6) were isolated from the leaves of Excoecaria agallocha. Their structures were elucidated by spectroscopic analyses. The new compound was characterized as $3 \beta-[(2 E, 4 E)-5$-oxo-decadienoyloxy]-olean-12-ene (1). Compounds $1-6$ were found inactive in vitro against several human cancer cell lines.

Key words Excoecaria agallocha; mangrove; pentacyclic triterpenoid

Being one of non-viviparous true mangrove species, $E x$ coecaria agallocha $\mathrm{L}$. (Euphorbiaceae) is naturally distributed on wetland along the coastlines in China, which has been used as a dart poison and fish poison in Southeastern Asia. Previous studies of this plant have characterized the constituents mainly as daphnane diterpene ester and labdane diterpenoids. ${ }^{1-6)}$ Some diterpenoids isolated have been found to possess anti-tumor promoting activity in vivo against mouse skin papillomas. ${ }^{7,8)}$ In screening for anti-tumor biologically active compounds from Chinese mangrove plants, we have isolated and elucidated a new pentacyclic triterpenoid (1), along with five known triterpenoids, $\beta$ amyrin acetate (2), ${ }^{9,10)}$ taraxerone (3), ${ }^{11,12)}$ 3-epitaraxerol (4), ${ }^{13,14)} 3$-epilupeol (5), ${ }^{14,15)}$ and taraxerol (6), ${ }^{11,12)}$ from the leaves of E. agallocha. This paper deals with the elucidation of the new triterpenoid using spectroscopic methods.

Compound $\mathbf{1}$ was obtained as a white needle crystal, which was found to be unsaturated triterpenoid from the positive Libermann-Buchard test. The HR-EI-MS gave a molecular ion peak at $\mathrm{m} / \mathrm{z} 590.4710$ corresponding to the molecular formula $\mathrm{C}_{40} \mathrm{H}_{62} \mathrm{O}_{3}$ (Calcd 590.4699). The IR spectrum exhibited conjugated carbonyl absorption bands at 1712 and $1695 \mathrm{~cm}^{-1}$. The UV spectrum showed maximum wavelength at $272 \mathrm{~nm}$ suggesting the presence of considerable conjugation in the molecule. The EI-MS spectrum was characteristic of the olean-12-ene series of triterpenoids. ${ }^{16}$ The major fragment at $\mathrm{m} / \mathrm{z} 218$ was due to retro-Diels-Alder cleavage of the $\mathrm{C}(12)-\mathrm{C}(13)$ bond, and which was accompanied by another fragment at $\mathrm{m} / \mathrm{z} 203$ resulting from the subsequent loss of a methyl group.

The ${ }^{1} \mathrm{H}$-NMR spectrum revealed the presence of eight methyl singlets at $\delta 0.84$ (Me-28), 0.88 (Me-29, 30), 0.90 (Me-24), 0.92 (Me-23), 0.98 (Me-25, 26), 1.14 (Me-27), one oxymethine proton at $\delta 4.61(\mathrm{t}, J=8 \mathrm{~Hz}, \mathrm{H}-3)$, and one olefinic proton at $\delta 5.19$ (br s, H-12). Moreover, in comparison with the ${ }^{13} \mathrm{C}$-NMR spectral data of compound 2 , the results were consistent with an $\beta$-amyrin type triterpene. The ${ }^{1} \mathrm{H}$-NMR spectrum showed four olefinic proton signals at $\delta$ 7.29 (dd, $\left.J=11.0,15.0 \mathrm{~Hz}, \mathrm{H}-3^{\prime}\right), 7.18$ (dd, $J=11.0,15.0 \mathrm{~Hz}$, H-4') 6.45 (d, $\left.J=15.0 \mathrm{~Hz}, \mathrm{H}-5^{\prime}\right)$, and $6.25(\mathrm{~d}, J=15.0 \mathrm{~Hz}, \mathrm{H}-$ $2^{\prime}$ ), which suggested the presence of trans-butadiene group. The ${ }^{13} \mathrm{C}$-NMR spectrum and DEPT experiment indicated two carbonyl group signals at $\delta 200.2\left(\mathrm{C}-5^{\prime}\right)$ and $165.7\left(\mathrm{C}-1^{\prime}\right)$. Together with the analysis of HMQC and HMBC, the sub- stituent group was identified as $(2 E, 4 E)$-5-oxo-decadienoyl, which was attached to an oxygen atom at C-3 of the molecule. Compound 1 was therefore determined as $3 \beta-[(2 E, 4 E)$ 5-oxo-decadienoyloxy]-olean-12-ene. This is the first report of its occurrence in nature.

Compounds 1-6 were evaluated for cytotoxic activity using MTT method. However, the results showed that all compounds were inactive $\left(\mathrm{IC}_{50}>50 \mu \mathrm{g} / \mathrm{ml}\right)$ against human cancer cell lines including lung adenocarcinoma (A549), stomach cancer (BGC-823), breast cancer (MCF-7), hepatoma (Bel7402), and human colon cancer (HCT-8) cell lines.

\section{Experimental}

General Experimental Procedures Melting points were determined on an XT-4 micro melting point apparatus and are uncorrected. Specific rotations were measured with Perkin-Elmer 241 polarimeter. IR spectra were recorded on a Nicolet Impact 400 FT-IR spectrophotometer. UV spectra were measured with a Shimadzu UV-260. NMR spectra were recorded in $\mathrm{CDCl}_{3}$ with INOVA $500 \mathrm{NMR}$ spectrometer, using visual $\mathrm{CDCl}_{3}$ resonances $\left({ }^{1} \mathrm{H} \delta 7.26,{ }^{13} \mathrm{C} \delta 77.0\right)$ for internal reference. EI-MS and HR-EI-MS were performed with an Autospec-Ultima ETOF spectrometer. Column chromatography was carried out on silica gel ( $300-400$ mesh). TLC analysis was carried out with glass precoated silica gel $\mathrm{GF}_{254}$ plates; and detection was performed by spraying with $5 \% \mathrm{H}_{2} \mathrm{SO}_{4}$ in ethanol, followed by heating at $110^{\circ} \mathrm{C}$ for $5 \mathrm{~min}$.

Plant Material The leaves of Excoecaria agallocha were collected at Guangxi province, P.R. China, in March 2005. A voucher specimen (No. 2005E06) was deposited in Institute of Materia Medica, Chinese Academy of Medical Sciences and Peking Union Medical College, P.R. China.

Extraction and Isolation Air-dried leaves of E. agallocha $(245 \mathrm{~g})$ were

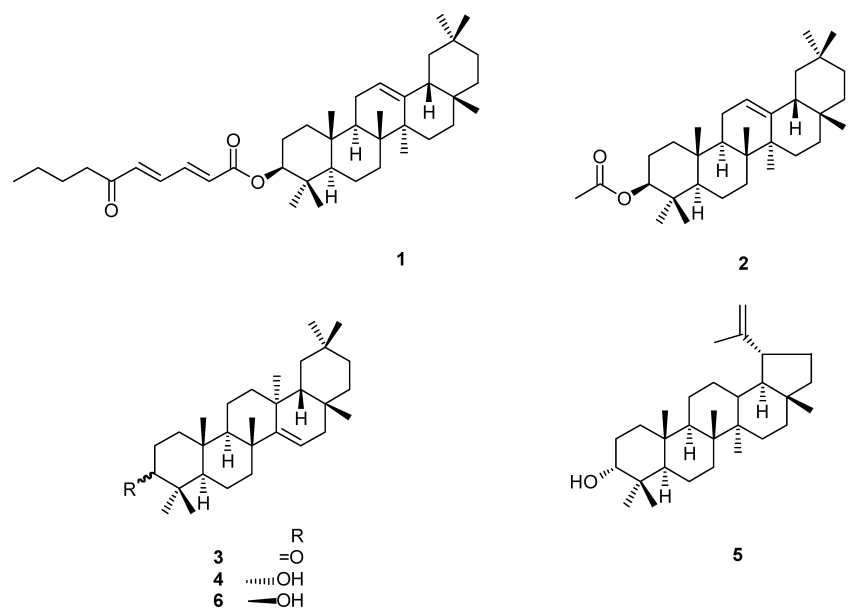


extracted with acetone $(31 \times 3)$ at room temperature for 1 month. The filtrate was concentrated under reduced pressure at $40^{\circ} \mathrm{C}$ to obtain the acetone extract $(20 \mathrm{~g})$. The extract was chromatographed over silica gel to afford 18 fractions, eluting with petroleum ether $\left(60-90{ }^{\circ} \mathrm{C}\right)$-acetone $(100: 0$ to $0: 100$, gradient increasing). The fractions $1-3(2 \mathrm{~g})$ were repeatedly purified by silica gel column chromatography using petroleum ether-acetone (100: 1), to yield compounds 1 (6.8 mg), 2 (106 mg), 3 (14 mg), 4 (57 mg), and $5(168 \mathrm{mg})$. The fractions 4 and $5(700 \mathrm{mg})$ were chromatographed over silica gel using petroleum ether-acetone $(98: 2)$ to afford compounds 6 (78 $\mathrm{mg})$, and $\beta$-sitosterol (41 mg).

$3 \beta$-[(2E,4E)-5-Oxo-decadienoyloxy]-olean-12-ene (1): White needle crystal (acetone); $\mathrm{mp} 186-188^{\circ} \mathrm{C} ;[\alpha]_{\mathrm{D}}^{23}+81.1^{\circ}\left(c=0.11, \mathrm{CHCl}_{3}\right) ; \mathrm{UV} \lambda_{\max }$ $(\mathrm{MeOH}) \mathrm{nm}(\log \varepsilon): 272$ (4.51); IR $(\mathrm{KBr}) \mathrm{cm}^{-1}: 2951,1712,1695,1601$, 1259, 1238, 1142, 1014, 1000; EI-MS $m / z$ (rel. int.) $590(\mathrm{M})^{+}(7), 408$ (6), 257 (5), 218 (100), 203 (72), 189 (31), 175 (10); HR-EI-MS m/z 590.4710 $(\mathrm{M})^{+}\left(\right.$Calcd for $\left.\mathrm{C}_{40} \mathrm{H}_{62} \mathrm{O}_{3}: 590.4699\right) ;{ }^{1} \mathrm{H}-\mathrm{NMR}\left(\mathrm{CDCl}_{3}\right) \delta: 7.29(1 \mathrm{H}$, dd, $\left.J=11.0,15.0 \mathrm{~Hz}, \mathrm{H}-3^{\prime}\right), 7.18\left(1 \mathrm{H}, \mathrm{dd}, J=11.0,15.0 \mathrm{~Hz}, \mathrm{H}-4^{\prime}\right), 6.45(1 \mathrm{H}, \mathrm{d}$, $\left.J=15.0 \mathrm{~Hz}, \mathrm{H}-5^{\prime}\right), 6.25\left(1 \mathrm{H}, \mathrm{d}, J=15.0 \mathrm{~Hz}, \mathrm{H}-2^{\prime}\right), 5.19$ (1H, br s, H-12), 4.61 $(1 \mathrm{H}, \mathrm{t}, J=8 \mathrm{~Hz}, \mathrm{H}-3), 2.60\left(2 \mathrm{H}, \mathrm{t}, J=7.5 \mathrm{~Hz}, \mathrm{H}-7^{\prime}\right), 1.61\left(2 \mathrm{H}, \mathrm{m}, \mathrm{H}-8^{\prime}\right), 1.36$ $\left(2 \mathrm{H}, \mathrm{m}, \mathrm{H}-9^{\prime}\right), 1.14$ (3H, s, Me-27), 0.98 (6H, s, Me-25, 26), 0.93 (3H, t, $\left.J=6.5 \mathrm{~Hz}, \mathrm{Me}-10^{\prime}\right), 0.92(3 \mathrm{H}, \mathrm{s}, \mathrm{Me}-23), 0.90(3 \mathrm{H}, \mathrm{s}, \mathrm{Me}-24), 0.88(6 \mathrm{H}, \mathrm{s}$, Me-29, 30), 0.88 (1H, overlapped, H-5), 0.84 (3H, s, Me-28); ${ }^{13} \mathrm{C}-\mathrm{NMR}$ $\left(\mathrm{CDCl}_{3}\right) \delta: 38.2(\mathrm{C}-1), 23.6(\mathrm{C}-2), 81.6(\mathrm{C}-3), 37.9(\mathrm{C}-4), 55.2(\mathrm{C}-5), 18.3$ (C-6), 32.6 (C-7), 39.8 (C-8), 47.6 (C-9), 36.8 (C-10), 23.5 (C-11), 121.6 (C-12), 145.2 (C-13), 41.7(C-14), 26.1 (C-15), 26.9 (C-16), 32.5 (C-17) 47.2 (C-18), 46.8 (C-19), 31.1 (C-20), 34.7 (C-21), 37.1 (C-22), 28.1 (C-23), 16.8 (C-24), 15.6 (C-25), 16.8 (C-26), 25.9 (C-27), 28.4 (C-28), 33.3 (C-29), 23.7 (C-30), $165.7\left(\mathrm{C}-1^{\prime}\right), 129.6\left(\mathrm{C}-2^{\prime}\right), 141.1\left(\mathrm{C}-3^{\prime}\right), 138.3\left(\mathrm{C}-4^{\prime}\right), 135.3$ (C-5'), $200.2\left(\mathrm{C}-6^{\prime}\right), 41.0\left(\mathrm{C}-7^{\prime}\right), 26.1\left(\mathrm{C}-8^{\prime}\right), 22.3\left(\mathrm{C}-9^{\prime}\right), 13.9\left(\mathrm{C}-10^{\prime}\right)$.

\section{References}

1) Ohhigashi H., Katsumata H., Kawazu K., Koshimizu K., Mitsui T., Agric. Biol. Chem., 38, 1093-1095 (1974).

2) Karalai C., Wiriyachitra P., Opfekuch H. J., Hecker E., Planta Med., 60, 351-355 (1994).

3) Konishi T., Azuma M., Itoga R., Kiyosawa S., Fujiwara Y., Shimada Y., Chem. Pharm. Bull., 44, 229-231 (1996).

4) Konishi T., Konoshima T., Fujiwara Y., Kiyosawa S., Chem. Pharm. Bull., 46, 721-722 (1998).

5) Anjaneyulu A. S. R., Lakshmana Rao V., Phytochemistry, 55, 891901 (2000).

6) Konishi T., Yamazoe K., Konoshima T., Maoka T., Fujiwara Y., Miyahara K., J. Nat. Prod., 66, 108-111 (2003).

7) Konishi T., Takasaki M., Tokuda H., Kiyosawa S., Konoshima T., Biol. Pharm. Bull., 21, 993-996 (1998).

8) Konoshima T., Konishi T., Takasaki M., Yamazoe K., Tokuda H., Biol. Pharm. Bull., 24, 1440-1442 (2001).

9) Ageta H., Arai Y., Phytochemistry, 22, 1801-1808 (1983).

10) Seo S., Tomita Y., Tori K., Tetrahedron Lett., 16, 7-10 (1975).

11) Bates R. B., Jacobsen N. E., Setzer W. N., Stessman C. C., Magn. Reson. Chem., 36, 539-541 (1998).

12) Sakurai N., Yaguchi Y., Inoue T., Phytochemistry, 26, 217-219 (1987).

13) Corbett R. E., Cumming S. D., Whitehead E. V., J. Chem.. Soc., Perkin Trans. I, 1972, 2827-2829 (1972).

14) Mahato S. B., Kundu A. P., Phytochemistry, 37, 1517-1575 (1994).

15) Puapairoj P., Naengchomnong W., Kijjoa A., Pinto M. M., Pedro M., Nascimento M. S. J., Silva A. M. S., Herz W., Planta Med., 71, 208213 (2005).

16) Shiojima K., Arai Y., Masuda K., Takase Y., Ageta T., Ageta H., Chem. Pharm. Bull., 40, 1683-1690 (1992). 